Relations industrielles

Industrial Relations

\title{
Harry Douglas Woods, 1907-1983
}

\section{Shirley B. Goldenberg et Gérard Hébert}

Volume 38, numéro 4, 1983

URI : https://id.erudit.org/iderudit/029400ar

DOI : https://doi.org/10.7202/029400ar

Aller au sommaire du numéro

Éditeur(s)

Département des relations industrielles de l'Université Laval

ISSN

0034-379X (imprimé)

1703-8138 (numérique)

Découvrir la revue

Citer cet article

Goldenberg, S. B. \& Hébert, G. (1983). Harry Douglas Woods, 1907-1983.

Relations industrielles / Industrial Relations, 38(4), 707-712.

https://doi.org/10.7202/029400ar

Tous droits réservés @ C Département des relations industrielles de l'Universite Laval, 1983
Ce document est protégé par la loi sur le droit d'auteur. L'utilisation des services d'Érudit (y compris la reproduction) est assujettie à sa politique d'utilisation que vous pouvez consulter en ligne.

https://apropos.erudit.org/fr/usagers/politique-dutilisation/ 


\title{
Harry Douglas Woods 1907-1983
}

\author{
Shirley B. Goldenberg \\ et
}

Gérard Hébert

With the death of H.D. Woods on August 7, 1983, the Canadian academic world lost a distinguished teacher, scholar and administrator. The loss will be particularly felt by the industrial relations community, of which he was the dean. He left us a rich legacy - his contributions to mediation and arbitration, his impact on policy formation in Canada and abroad, his influence on generations of students, his reports to government and his classic work on Labour Policy. These are a living monument to his memory, as is the Industrial Relations Centre he founded at McGill. Those of us who had the privilege to know him as a friend and colleague will remember him with affection as a kind and generous human being.

Harry Douglas Woods, O.C., M.A., LL.D., F.R.S.C., better known to most of us as 'Bus', was born in New Brunswick on January 16, 1907. He received a B.A. from the University of New Brunswick, an M.A. from McGill, and did graduate studies at the London School of Economics and the University of Toronto. His teaching career, which began in 1937 at United College in Winnipeg, was interrupted by the War. He spent the war years in the army. Following his military service, he received an appointment at McGill where he taught until 1978. He then continued his academic career as a visiting lecturer at the University of New Brunswick until his death.

Bus Woods occupied various administrative positions at McGill, in addition to his primary task as a professor. He served, in turn, as Director of the School of Commerce, Director of the Industrial Relations Centre, Dean of the Faculty of Arts and Science and Director of the M.B.A. Program in the Faculty of Management. His term as Dean of Arts and Science (1964-69) coincided with the period of serious student unrest. He guided the Faculty, and the University, through that period with the patience, wisdom and understanding that made him a successful mediator and arbitrator.

Bus Woods' ability in the field of industrial relations was recognized throughout Canada. He was a founding member of the Canadian Industrial Relations Research Institute (since renamed the

Relat. ind., vol. 38, no 4, 1983 ๑ PUL ISSN 0034-379 X 
Canadian Industrial Relations Association) and served as its first president. When that organization established an annual award of distinction in 1980, Bus Woods was the first recipient of the honour. It was an appropriate choice. Governments of various provinces called on him for advice. He was a consultant to the Committee on Manitoba's Economic Future, Chairman of the Joint Labour Management Committee of Manitoba and consultant on industrial relations to the government of New Brunswick.

His most important public assignment was in 1966, when Lester Pearson, then Prime Minister of Canada, appointed him Chairman of a Task Force on Labour Relations. The 'Woods Task Force', as it came to be known, conducted the most comprehensive study of labour relations ever undertaken in Canada. It commissioned over a hundred studies by scholars in different disciplines. About a third of the studies were published, contributing significantly to the literature in the field. The recommendations of the Task Force had an important impact on labour relations policy throughout Canada.

Bus Woods' reputation, and his contributions, extended beyond Canada. Appointed by the ILO, he served as an expert advisor on industrial relations in the Phillippines. The Ministry of Labour of Jamaica selected him to deal with a major industrial dispute. He was the only Canadian to serve as president of the National Academy of Arbitrators, a prestigous American organization.

His achievements brought him numerous honours. McGill made him a Professor Emeritus on his retirement. The University of New Brunswick awarded him a LL.D., as a distinguished alumnus. He was elected a Fellow of the Royal Society of Canada and named an Officer of the Order of Canada. But if Bus Woods had been asked to choose the tribute that touched him the most, it would have been the one he received from his students. If he had been asked to name the aspect of his career that had given him the greatest pleasure, it would undoubtedly have been his teaching. He loved young people and they loved him. When his students learned, with sorrow, of his impending retirement from McGill, they gave him a farewell party. It was a moving event. He treasured the plaque that was presented to him. The inscription on that plaque reads as follows: 
To H.D. Woods

Professor of Industrial Relations

at McGill

From your students, with much respect and

in appreciation for

your dedicáied work.

These words express the feelings, not only of his students, but of all of us who were privileged to know him.

Shirley B. Goldenberg

McGill University

La communauté canadienne de relations industrielles vient de perdre un de ses piliers. Harry Douglas Woods est décédé d'un cancer à l'hôpital civique d'Ottawa le 7 août dernier. Encore actif, il était à Ottawa pour y effectuer une analyse des activités du Conseil canadien des relations du travail en rapport avec les objectifs $d u$ Rapport Woods, publié en 1968, quand il y fut terrassé par une paralysie, dont les suites devaient l'emporter peu de temps après.

Grand et fort comme un de ces ormes qui bordent la rivière SaintJean, au Nouveau-Brunswick, sa patrie d'origine et de retraite, il avait aussi le sourire et l'oeil malicieux de ses aïeux d'Irlande et d'Angleterre. Sa bonhomie lui avait valu d'être connu de tous par son surnom familier de Bus Woods, surnom d'origine peu connue, qui évoquait ses multiples activités. Atńlète et sportif, c'est au cours d'une joute dans l'Ouest canadien qu'il avait gagné le coeur de celle qui devint son épouse et son inspiration tout au long de sa vie.

H.D. Woods avait la trempe des pionniers. Après avoir fait la guerre, il revint à l'Université McGill, d'où il avait obtenu une maîtrise en 1931. Dès 1948, il y fonde le Centre de relations industrielles, dont il demeura le directeur jusqu'à la fin des années 1960. Quelques années plus tard, il organisa un premier congrès de relations industrielles, congrès qui se répète à chaque année depuis lors avec beaucoup de succès. En 1962, il publia, avec Sylvia Ostry, le premier volume de caractère universitaire sur les relations industrielles au Canada, Labour Economics and Labour Policy in Canada. Peu après, il fonda, avec l'abbé Gérard Dion de l'Université Laval, l'Institut 
canadien de recherche en relations industrielles, qui constitue aujourd'hui, sous un nom légèrement modifié, une des sociétés savantes actives dans le domaine des sciences sociales.

Le professeur Woods concevait son rôle d'universitaire comme débordant bien au-delà du campus. La représentation multipartite $q u$ 'il introduisit dans la structure du Centre de relations industrielles en est un signe parmi bien d'autres. En dehors de l'université, Woods était connu comme un conciliateur particulièrement habile et un arbitre estimé. Sa préoccupation de rechercher et de promouvoir les politiques de relations industrielles qu'il croyait les plus aptes à développer une véritable démocratie industrielle se retrouve autant dans le titre de son ouvrage principal, Labour Policy in Canada, que dans sa participation à de nombreux comités et commissions publiques, en particulier le Groupe de travail sur les relations du travail au Canada dont il dirigea les nombreuses activités de 1966 à 1968.

Toute sa vie, Woods fut un conciliateur extraordinaire. Même quand il occupait une fonction d'arbitre, il essayait, par tous les moyens à sa disposition, d'obtenir que les parties s'entendent entre elles plutôt que de leur imposer la solution que lui-même aurait pu considérer la plus juste. En fait, il recherchait bien davantage les solutions acceptables aux parties que les mesures justes et équitables qu'il aurait pu proposer de lui-même. Il avait quelque difficulté à voir l'utilité de concepts abstraits comme le juste salaire et le juste profit. Ses qualités remarquables en la matière lui ont valu d'être le premier canadien à occuper la présidence de la prestigieuse National Academy of Arbitrators des États-Unis.

Ses talents de conciliateur lui ont été d'une aide précieuse au moment où il fut doyen de la Faculté des arts et des sciences, de 1965 à 1970. Cette période correspond à celle où tous les campus universitaires furent secoués par l'agitation de mouvements étudiants turbulents. Woods a permis à l'Université McGill de traverser ces difficultés tout en évitant les incidents fâcheux que d'autres institutions nord-américaines ont malheureusement connus.

Le prestige dont a joui H.D. Woods, tout au long de sa carrière, lui venait autant de ses qualités humaines que de ses aptitudes intellectuelles et professionnelles. Comme professeur, ses étudiants recherchaient dans ses cours les intuitions brillantes qu'il avait sur les problèmes contemporains de relations de travail. Par contre, ces intuitions ne se présentaient pas toujours dans un ordre très rigoureux; il fallait demeurer attentif pour les dégager de développements parfois 
sinueux. Ses écrits manifestaient des qualités qui correspondaient à ses aptitudes particulières. Ainsi, les pages qu'il a écrites sur la conciliation-médiation et l'arbitrage sont particulièrement éclairantes. La distinction entre approche accommodative et approche normative, qu'il a été le premier à exposer avec tant de clarté, fait encore partie du bagage nécessaire des connaissances tant des étudiants que des praticiens. D'un autre côté, ses écrits n'ont ni l'abondance ni la rigueur d'autres universitaires. Dans les nombreux comités et commissions auxquels il a participé ou qu'il a dirigés, il avait le don d'animer les discussions et d'orienter les conclusions, mais il n'était pas toujours celui qui consacrait le plus de temps à la rédaction du rapport final. Il s'est avéré à la fois un universitaire préoccupé des aspects pratiques des problèmes qu'il étudiait, et un praticien sans cesse à la recherche de formules nouvelles à communiquer aux divers auditoires auxquels il s'adressait. Cet aspect de sa personnalité n'a pas peu contribué au rayonnement énorme qu'il a eu, tant au Canada qu'aux États-Unis, et même, à l'occasion de certaines missions extérieures, dans différents pays du monde.

Depuis la guerre et jusqu'à sa retraite vers 1975, l'Université McGill a toujours été au coeur des activités de Bus Woods. C'est donc dire que Montréal a été son premier champ d'action. Le Centre de relations industrielles de McGill comptait parmi ses membres plusieurs canadiens français. Ses qualités de conciliateur et d'arbitre l'ont amené à exercer ces fonctions dans plusieurs conflits où la majorité des intervenants étaient de langue française. Pourtant, Bus Woods n'a jamais parlé français, et il le comprenait difficilement. Cependant, il demeure un des anglo-canadiens qui a le mieux compris les conflits de travail du Québec. L'auteur de ces lignes l'a entendu s'exclamer, au sortir de rencontres auxquelles participaient des représentants syndicaux et universitaires des deux grandes cultures canadiennes: "Ils ne comprendront rien à la situation du Québec, ces anglais de Toronto!» Son rôle de conciliateur dans la première grève de cadres au Québec, celle des réalisateurs de Radio-Canada en 1959, a été efficace, même si les sessions se sont déroulées en anglais, alors que patrons et syndiqués étaient tous de langue française: les parties n'avaient réussi à s'entendre sur aucun autre nom comme conciliateur que sur celui de Woods. Le professeur Woods a lui-même offert au soussigné de présenter sa thèse de doctorat à l'Université McGill en français; il était à la fois amusant et édifiant de voir ce grand anglais parcourir les chapitres de cette thèse en devenir, le dictionnaire français-anglais à portée de la main sur le coin de son pupitre. 
Si Bus Woods a eu tellement de succès dans ses nombreuses activités et s'il a inspiré tant de personnes oeuvrant dans divers domaines, cela est dû, outre ses qualités intellectuelles, à son dynamisme et à sa personnalité attachante. Il était particulièrement fidèle envers ceux qu'il estimait et qu'il aimait. Ses nombreux amis se souviennent de lui comme d'un compagnon agréable, parfois déconcertant, rempli de multiples intérêts bien au-delà des relations de travail, y compris tous les sports qu'il adorait. Romantique et déconcertant, il l'a été jusque dans ses dernières volontés, comme s'il avait voulu se payer un dernier bon tour aux dépens de ceux qu'il aimait le plus.

La contribution de H.D. Woods aux études universitaires en relations industrielles a tenu bien davantage à l'entraînement qu'il a su imprimer à tous ceux qui oeuvraient dans le domaine qu'aux ouvrages qu'il laisse à la postérité, même si ceux-ci ne sont pas négligeables, en particulier dans le domaine de la conciliation et des politiques publiques en matière de relations de travail. À tous ceux qui l'ont connu de plus près, il laisse le souvenir d'un personnage fort, dynamique, mais aussi sensible et profondément humain, dans tous les aspects que ce terme peut évoquer. D'autres laisseront une oeuvre écrite plus impressionnante, bien peu laisseront le souvenir d'une personnalité aussi attachante.

Université de Montréal

Gérard Hébert 